\title{
A class of nonlocal problems of fractional differential equations with composition of derivative and parameters
}

\author{
Mei Jia ${ }^{1 *}$, Lin Li ${ }^{1}$, Xiping Liu', Junqiu Song ${ }^{1}$ and Zhanbing Bai
}

\section{"Correspondence:}

jiamei-usst@163.com

${ }^{1}$ College of Science, University of

Shanghai for Science and

Technology, Shanghai, China

Full list of author information is

available at the end of the article

\section{严 Springer}

\begin{abstract}
In this paper, we study existence and nonexistence of positive solutions for a class of Riemann-Stieltjes integral boundary value problems of fractional differential equations with parameters. By using the fixed point index theory, some new sufficient conditions for the existence of at least one, two and the nonexistence of positive solutions are obtained. The results we obtain show the influence of parameter $\lambda$ and parameter $a$ on the existence of positive solutions. Finally, some examples are given to illustrate our main results.
\end{abstract}

MSC: 34A08; 34B09; 34B18

Keywords: Riemann-Liouville fractional derivative; Riemann-Stieltjes integral boundary conditions; Disturbance parameter; Fixed point theorem

\section{Introduction}

In this paper, we investigate existence and nonexistence of positive solutions for a class of Riemann-Stieltjes integral boundary value problems of fractional differential equations with parameters

$$
\left\{\begin{array}{l}
D_{0^{+}}^{\alpha}\left(p(t) D_{0^{+}}^{\beta} u(t)\right)+\lambda f(t, u(t))=0, \quad t \in(0,1) \\
\lim _{t \rightarrow 0^{+}} t^{2-\beta} u(t)=a, \quad u(1)=\int_{0}^{1} u(s) \mathrm{d} A(s) \\
\lim _{t \rightarrow 0^{+}} t^{1-\alpha} p(t) D_{0^{+}}^{\beta} u(t)=0
\end{array}\right.
$$

where $D_{0^{+}}^{\alpha}$ and $D_{0^{+}}^{\beta}$ are the Riemann-Liouville fractional derivatives with $0<\alpha \leq 1,1<$ $\beta \leq 2$. The parameters $\lambda>0, a \geq 0, p \in C([0,1],(0,+\infty)), f:[0,1] \times[0,+\infty) \rightarrow[0,+\infty)$ are given functions, and $f$ may be discontinuous but satisfies the $L^{q}$-Carathéodory conditions. $\int_{0}^{1} u(s) \mathrm{d} A(s)$ denotes the Riemann-Stieltjes integral with respect to $A$.

By using the fixed point index theory, some new sufficient conditions for the existence of at least one, two and the nonexistence of positive solutions are obtained. The theorems we obtain show the influence of parameter $\lambda$ and parameter $a$ on the existence of positive solutions.

In recent decades, with the wide applications of fractional differential equations in physics, engineering, biology, chemistry, and many other fields, researchers have been

(c) The Author(s) 2019. This article is distributed under the terms of the Creative Commons Attribution 4.0 International License (http://creativecommons.org/licenses/by/4.0/), which permits unrestricted use, distribution, and reproduction in any medium, provided you give appropriate credit to the original author(s) and the source, provide a link to the Creative Commons license, and indicate if changes were made. 
paying more and more attention to them, see [1-12] and the references therein. At the same time, many problems of fluid mechanics, bioengineering, chemical engineering, and so on could be attributed to the integral boundary value problems, which are nonlocal problems. Therefore, a lot of meaningful research results have been obtained, see [13-20] and the references therein. The eigenvalue problem is a relatively active part of the differential equation theory, and there have been many results, see [21-30] and the references therein. Nowadays, when solving many practical problems, there will inevitably be errors and those errors will often affect the existence of the solution to a large extent. Therefore, it is meaningful to study the boundary value problem of fractional differential equations with disturbance parameters, see [31-35] and the references therein.

As a generalization of classical Riemann integral, Riemann-Stieltjes integral boundary value problem has a stronger applicability, which not only contains the classical Riemann integral boundary value problem, but also includes two-point boundary value and multipoint boundary value. In this paper, we investigate existence and nonexistence of positive solutions for a class of Riemann-Stieltjes integral boundary value problems of fractional differential equations with parameters (1.1).

The paper is organized as follows. In Sect. 2, we present some necessary definitions and lemmas which will be used to prove our main results. We study the properties of integral kernels and obtain inequalities about the integral kernels. We prove the complete continuity of operators. In Sect. 3, we investigate the existence of at least one positive solution for boundary value problem (1.1). In Sect. 4, sufficient conditions for the existence of at least two positive solution of boundary value problem (1.1) and the nonexistence of positive solution of boundary value problem (1.1) are established. In Sect. 5, we give some examples to illustrate our main result.

Throughout this paper, we assume that $A(t)$ is a monotone increasing function, $\int_{0}^{1} s^{\beta-2} \mathrm{~d} A(s)$ exists, and

$$
1-\int_{0}^{1} s^{\beta-1} \mathrm{~d} A(s)>0
$$

$f$ satisfies the $L^{q}$-Carathéodory conditions, that is,

(1) $f(\cdot, u)$ is measurable for all $u \in[0,+\infty)$;

(2) $f(t, \cdot)$ is continuous for a.e. $t \in[0,1]$;

(3) for every $r>0$, there exists $\varphi_{r} \in L^{q}[0,1]$ such that

$$
\left|f\left(t, t^{\beta-2} u\right)\right| \leq \varphi_{r}(t) \quad \text { for all } u \in[0, r] \text { and a.e. } t \in[0,1]
$$

where $q>\frac{1}{\alpha}$ if $0<\alpha<1$ and $q=1$ if $\alpha=1$.

For $L^{q}[0,1]$, we denote the norm $\|\varphi\|_{L^{q}}=\left(\int_{0}^{1}|\varphi(t)|^{q} \mathrm{~d} t\right)^{\frac{1}{q}}$.

\section{Preliminaries}

The definitions of fractional integral and fractional derivative and the related lemmas can be found in $[3,4]$. 
Lemma 2.1 (See [3], Theorem 2.4 and [4], Lemma 2.5) Let $p>0$ and $n=\lceil p\rceil=\min \{z \in$ $\mathbb{Z}: z \geq p\}$. If $u \in L^{1}[0,1]$ and $I_{0^{+}}^{n-p} u \in A C^{n}[0,1]$, then the equality

$$
I_{0^{+}}^{p}\left(D_{0^{+}}^{p} u\right)(t)=u(t)-\sum_{k=1}^{n} c_{k} t^{p-k}
$$

holds a.e. on $[0,1]$.

Lemma 2.2 If $0<\alpha<1, D_{0^{+}}^{\alpha} u \in L^{1}[0,1]$ and $\lim _{t \rightarrow 0^{+}} t^{1-\alpha} u(t)=c$, where $c$ is a constant, then $I_{0^{+}}^{1-\alpha} u \in A C^{1}[0,1]$.

Proof Since $\lim _{t \rightarrow 0^{+}} t^{1-\alpha} u(t)=c$, then for any $\varepsilon>0$, there exists a constant $\delta>0$ such that $\left|t^{1-\alpha} u(t)-c\right|<\frac{\varepsilon}{\Gamma(\alpha)}$ whenever $0<t<\delta$, and

$$
\begin{aligned}
\left|I_{0^{+}}^{1-\alpha} u(t)-c \Gamma(\alpha)\right| & =\left|I_{0^{+}}^{1-\alpha} u(t)-c I_{0^{+}}^{1-\alpha} t^{\alpha-1}\right| \\
& \leq \frac{1}{\Gamma(1-\alpha)} \int_{0}^{t}(t-s)^{-\alpha}\left|u(s)-c s^{\alpha-1}\right| \mathrm{d} s \\
& =\frac{1}{\Gamma(1-\alpha)} \int_{0}^{t}(t-s)^{-\alpha} s^{\alpha-1}\left|s^{1-\alpha} u(s)-c\right| \mathrm{d} s \\
& <\varepsilon .
\end{aligned}
$$

Hence, we have $\lim _{t \rightarrow 0^{+}} I_{0^{+}}^{1-\alpha} u(t)=c \Gamma(\alpha)$.

Let $\phi(t)=D_{0^{+}}^{\alpha} u(t)=\frac{\mathrm{d}}{\mathrm{d} t} I_{0^{+}}^{1-\alpha} u(t)$, then $\phi \in L^{1}[0,1]$ and

$$
I_{0^{+}}^{1-\alpha} u(t)=c \Gamma(\alpha)+\int_{0}^{t} \phi(s) \mathrm{d} s
$$

Therefore, $I_{0^{+}}^{1-\alpha} u \in A C^{1}[0,1]$.

Let

$$
E:=C_{2-\beta}[0,1]=\left\{u \in C(0,1]: t^{2-\beta} u(t) \in C[0,1]\right\},
$$

then $E$ is a Banach space with the norm $\|u\|=\sup _{t \in[0,1]} t^{2-\beta}|u(t)|$.

Definition 2.1 A function $u=u(t)$ is called a solution of fractional boundary value problem (1.1) if $u \in E$ and satisfies (1.1). Furthermore, $u=u(t)$ is called a positive solution of fractional boundary value problem (1.1) if $u(t)>0, t \in(0,1)$.

Lemma 2.3 For any $y \in L^{q}[0,1]$, the fractional differential initial value problem

$$
\left\{\begin{array}{l}
D_{0^{+}}^{\alpha} \nu(t)+y(t)=0, \quad t \in(0,1), \\
\lim _{t \rightarrow 0^{+}} t^{1-\alpha} v(t)=0
\end{array}\right.
$$

has a unique solution

$$
v(t)=-\frac{1}{\Gamma(\alpha)} \int_{0}^{t}(t-s)^{\alpha-1} y(s) \mathrm{d} s .
$$


Proof Suppose that $v=v(t)$ is a solution of initial value problem (2.1). Since $y \in L^{q}[0,1]$, then $D_{0^{+}}^{\alpha} v \in L^{1}[0,1]$. Because $\lim _{t \rightarrow 0^{+}} t^{1-\alpha} v(t)=0$, it follows $I_{0^{+}}^{1-\alpha} v \in A C^{1}[0,1]$ from Lemma 2.2. Thus, by Lemma 2.1, we have

$$
v(t)=-I_{0^{+}}^{\alpha} y(t)+c_{1} t^{\alpha-1}
$$

The initial condition $\lim _{t \rightarrow 0^{+}} t^{1-\alpha} v(t)=0$ implies that $c_{1}=0$. Thus,

$$
v(t)=-\frac{1}{\Gamma(\alpha)} \int_{0}^{t}(t-s)^{\alpha-1} y(s) \mathrm{d} s .
$$

On the other hand, if $v=v(t)$ satisfies (2.2), we can easily show that $v$ satisfies the equation of initial value problem (2.1).

Next, we show that $\lim _{t \rightarrow 0^{+}} t^{1-\alpha} v(t)=0$.

Let

$$
\phi_{1}(t)=\left\{\begin{array}{ll}
t^{\alpha-1}, & 0<t \leq 1, \\
0, & \text { else }
\end{array} \quad \text { and } \quad \phi_{2}(t)= \begin{cases}y(t), & 0 \leq t \leq 1 \\
0, & \text { else }\end{cases}\right.
$$

$F(t)$ is given by the convolution form, that is,

$$
F(t)=\left(\phi_{1} * \phi_{2}\right)(t)=\int_{-\infty}^{+\infty} \phi_{1}(t-s) \phi_{2}(s) \mathrm{d} s
$$

If $0<\alpha<1$, since $q>\frac{1}{\alpha}$, we have $\frac{q(\alpha-1)}{q-1}>-1$ and $\phi_{1} \in L^{\frac{q}{q-1}}(\mathbb{R})$. Hence,

$$
\lim _{\Delta t \rightarrow 0^{+}} \int_{\mathbb{R}}\left|\phi_{1}(s+\Delta t)-\phi_{1}(s)\right|^{\frac{q}{q-1}} \mathrm{~d} s=0 .
$$

In view of $\phi_{2} \in L^{q}(\mathbb{R})$, we can get that

$$
\begin{aligned}
|F(t+\Delta t)-F(t)| & =\left|\int_{\mathbb{R}} \phi_{1}(t+\Delta t-s) \phi_{2}(s) \mathrm{d} s-\int_{\mathbb{R}} \phi_{1}(t-s) \phi_{2}(s) \mathrm{d} s\right| \\
& \leq \int_{\mathbb{R}}\left|\phi_{1}(t+\Delta t-s)-\phi_{1}(t-s)\right|\left|\phi_{2}(s)\right| \mathrm{d} s \\
& \leq\left(\int_{\mathbb{R}}\left|\phi_{1}(s+\Delta t)-\phi_{1}(s)\right|^{\frac{q}{q-1}} \mathrm{~d} s\right)^{\frac{q-1}{q}}\left(\int_{\mathbb{R}}\left|\phi_{2}(s)\right|^{q} \mathrm{~d} s\right)^{\frac{1}{q}} \\
& \leq\|y\|_{L^{q}}\left(\int_{\mathbb{R}}\left|\phi_{1}(s+\Delta t)-\phi_{1}(s)\right|^{\frac{q}{q-1}} \mathrm{~d} s\right)^{\frac{q-1}{q}} \\
& \rightarrow 0 \quad(\Delta t \rightarrow 0) .
\end{aligned}
$$

If $\alpha=1$, it is obvious that $|F(t+\Delta t)-F(t)| \rightarrow 0(\Delta t \rightarrow 0)$.

Hence, $F(t)$ is uniformly continuous on $\mathbb{R}$, then we can get that

$$
\lim _{t \rightarrow 0} t^{1-\alpha} F(t)=0
$$


Because

$$
F(t)=v(t)=\int_{0}^{t}(t-s)^{\alpha-1} h(s) \mathrm{d} s, \quad 0 \leq t \leq 1
$$

Then we have $\lim _{t \rightarrow 0^{+}} t^{1-\alpha} v(t)=0$.

Remark 2.1 For any $y \in L^{q}[0,1], v=v(t)$, which satisfies (2.2), is uniformly continuous on $[0,1]$.

For convenience, we denote

$$
g(t)=\left(1-\int_{0}^{1} s^{\beta-1} \mathrm{~d} A(s)\right)^{-1}\left(\int_{0}^{1} s^{\beta-2} \mathrm{~d} A(s)-1\right) t^{\beta-1}+t^{\beta-2} .
$$

Lemma 2.4 For any $h \in C[0,1]$, the integral boundary value problem of linear fractional differential equation

$$
\left\{\begin{array}{l}
D_{0^{+}}^{\beta} u(t)=h(t), \quad t \in(0,1), \\
\lim _{t \rightarrow 0^{+}} t^{2-\beta} u(t)=a, \quad u(1)=\int_{0}^{1} u(s) \mathrm{d} A(s)
\end{array}\right.
$$

has a unique solution

$$
u(t)=-\int_{0}^{1} G_{1}(t, s) h(s) \mathrm{d} s+a g(t)
$$

where

$$
\begin{aligned}
& G_{1}(t, s)=K(t, s)+\frac{t^{\beta-1}}{1-\int_{0}^{1} s^{\beta-1} \mathrm{~d} A(s)} \int_{0}^{1} K(\tau, s) \mathrm{d} A(\tau), \\
& K(t, s)=\frac{1}{\Gamma(\beta)}\left\{\begin{array}{l}
t^{\beta-1}(1-s)^{\beta-1}-(t-s)^{\beta-1}, \quad 0 \leq s<t \leq 1, \\
t^{\beta-1}(1-s)^{\beta-1}, \quad 0 \leq t \leq s \leq 1 .
\end{array}\right.
\end{aligned}
$$

Proof Suppose that $u=u(t)$ is a solution of boundary value problem (2.5). Since $h \in$ $C[0,1]$, then $I_{0^{+}}^{2-\beta} u \in A C^{2}[0,1]$. Thus, by Lemma 2.1 , we have

$$
u(t)=I_{0^{+}}^{\beta} h(t)+c_{1} t^{\beta-1}+c_{2} t^{\beta-2} .
$$

The boundary condition $\lim _{t \rightarrow 0^{+}} t^{2-\beta} u(t)=a$ implies that $c_{2}=a$. Then

$$
u(t)=I_{0^{+}}^{\beta} h(t)+c_{1} t^{\beta-1}+a t^{\beta-2} .
$$

Hence

$$
u(1)=\frac{1}{\Gamma(\beta)} \int_{0}^{1}(1-s)^{\beta-1} h(s) \mathrm{d} s+c_{1}+a,
$$




$$
\begin{aligned}
\int_{0}^{1} u(s) \mathrm{d} A(s)= & \frac{1}{\Gamma(\beta)} \int_{0}^{1}\left(\int_{0}^{s}(s-\tau)^{\beta-1} h(\tau) \mathrm{d} \tau\right) \mathrm{d} A(s) \\
& +c_{1} \int_{0}^{1} s^{\beta-1} \mathrm{~d} A(s)+a \int_{0}^{1} s^{\beta-2} \mathrm{~d} A(s) .
\end{aligned}
$$

By the boundary condition $u(1)=\int_{0}^{1} u(s) \mathrm{d} A(s)$, we obtain that

$$
\begin{aligned}
c_{1}= & \frac{1}{1-\int_{0}^{1} s^{\beta-1} \mathrm{~d} A(s)}\left(\frac{1}{\Gamma(\beta)} \int_{0}^{1}\left(\int_{0}^{s}(s-\tau)^{\beta-1} h(\tau) \mathrm{d} \tau\right) \mathrm{d} A(s)\right. \\
& \left.-\frac{1}{\Gamma(\beta)} \int_{0}^{1}(1-s)^{\beta-1} h(s) \mathrm{d} s+a \int_{0}^{1} s^{\beta-2} \mathrm{~d} A(s)-a\right) .
\end{aligned}
$$

Substituting $c_{1}$ and $c_{2}$ into (2.9), we can get that

$$
\begin{aligned}
u(t)= & \frac{1}{\Gamma(\beta)} \int_{0}^{t}(t-s)^{\beta-1} h(s) \mathrm{d} s+c_{1} t^{\beta-1}+c_{2} t^{\beta-2} \\
= & \frac{1}{\Gamma(\beta)} \int_{0}^{t}(t-s)^{\beta-1} h(s) \mathrm{d} s \\
& +\frac{t^{\beta-1}}{1-\int_{0}^{1} s^{\beta-1} \mathrm{~d} A(s)}\left(\frac{1}{\Gamma(\beta)} \int_{0}^{1}\left(\int_{0}^{s}(s-\tau)^{\beta-1} h(\tau) \mathrm{d} \tau\right) \mathrm{d} A(s)\right. \\
& \left.-\frac{1}{\Gamma(\beta)} \int_{0}^{1}(1-s)^{\beta-1} h(s) \mathrm{d} s+a \int_{0}^{1} s^{\beta-2} \mathrm{~d} A(s)-a\right)+a t^{\beta-2} \\
= & \frac{1}{\Gamma(\beta)} \int_{0}^{t}(t-s)^{\beta-1} h(s) \mathrm{d} s \\
& -\left(\frac{t^{\beta-1}}{\Gamma(\beta)} \int_{0}^{1}(1-s)^{\beta-1} h(s) \mathrm{d} s+\frac{t^{\beta-1} \int_{0}^{1} \tau^{\beta-1} \mathrm{~d} A(\tau) \int_{0}^{1}(1-s)^{\beta-1} h(s) \mathrm{d} s}{\Gamma(\beta)\left(1-\int_{0}^{1} s^{\beta-1} \mathrm{~d} A(s)\right)}\right) \\
& +\frac{t^{\beta-1} \int_{0}^{1}\left(\int_{s}^{1}(\tau-s)^{\beta-1} \mathrm{~d} A(\tau)\right) h(s) \mathrm{d} s}{\Gamma(\beta)\left(1-\int_{0}^{1} s^{\beta-1} \mathrm{~d} A(s)\right)}+\frac{a t^{\beta-1}\left(\int_{0}^{1} s^{\beta-2} \mathrm{~d} A(s)-1\right)}{1-\int_{0}^{1} s^{\beta-1} \mathrm{~d} A(s)}+a t^{\beta-2} \\
= & -\int_{0}^{1}\left(K(t, s)+\frac{t^{\beta-1}}{1-\int_{0}^{1} s^{\beta-1} \mathrm{~d} A(s)} \int_{0}^{1} K(\tau, s) \mathrm{d} A(\tau)\right) h(s) \mathrm{d} s+a g(t) \\
& \int_{0}^{1}(t, s) h(s) \mathrm{d} s+a g(t) . \\
& \frac{1}{\Gamma}(\tau)
\end{aligned}
$$

On the other hand, if $u$ satisfies (2.6), then $u$ satisfies (2.9), too. It follows from (2.9)

$$
D_{0^{+}}^{\beta} u(t)=D_{0^{+}}^{\beta} I_{0^{+}}^{\beta} h(t)=D^{2}\left(I_{0^{+}}^{2-\beta} I_{0^{+}}^{\beta} h\right)(t)=h(t),
$$

which implies that the equation of boundary value problem (2.5) is satisfied.

We can easily show that $u$ satisfies the boundary conditions of boundary value problem (2.5).

Lemma 2.5 If $u \in E$, then boundary value problem (1.1) is equivalent to the following integral equation:

$$
u(t)=\lambda \int_{0}^{1} G(t, s) f(s, u(s)) \mathrm{d} s+a g(t)
$$


where

$$
G(t, s)=\frac{1}{\Gamma(\alpha)} \int_{s}^{1} \frac{1}{p(\tau)} G_{1}(t, \tau)(\tau-s)^{\alpha-1} \mathrm{~d} \tau .
$$

Proof Let $u=u(t)$ be a solution of boundary value problem (1.1) and denote $v(t)=$ $p(t) D_{0^{+}}^{\beta} u(t), y(t)=\lambda f(t, u(t)), h(t)=\frac{v(t)}{p(t)}$. By Lemma 2.3 and Lemma 2.4, we have

$$
u(t)=\lambda \int_{0}^{1} \frac{1}{p(s)} G_{1}(t, s) \int_{0}^{s} \frac{(s-\tau)^{\alpha-1}}{\Gamma(\alpha)} f(\tau, u(\tau)) \mathrm{d} \tau \mathrm{d} s+a g(t) .
$$

By exchanging integral order, we can get that

$$
\begin{aligned}
u(t) & =\lambda \int_{0}^{1} \frac{1}{\Gamma(\alpha)} f(s, u(s)) \int_{s}^{1} \frac{1}{p(\tau)} G_{1}(t, \tau)(\tau-s)^{\alpha-1} \mathrm{~d} \tau \mathrm{d} s+a g(t) \\
& =\lambda \int_{0}^{1} G(t, s) f(s, u(s)) \mathrm{d} s+a g(t),
\end{aligned}
$$

where $G(t, s)$ is defined by $(2.11)$.

On the other hand, if $u$ satisfies (2.10), the $u$ will also satisfy (2.12). By Lemma 2.3 and Lemma 2.4, $u$ satisfies boundary value problem (1.1).

Denote constants

$$
\begin{aligned}
& m_{i}=\int_{0}^{1} s^{\beta-i} \mathrm{~d} A(s), \quad i=0,1,2, \\
& \gamma_{0}=\frac{(\beta-1)\left(m_{1}-m_{0}\right)}{1-m_{1}+m_{2}},
\end{aligned}
$$

and the function

$$
g_{1}(s)=\frac{1}{\Gamma(\alpha) \Gamma(\beta)\left(1-m_{1}\right)} \int_{s}^{1} \frac{\tau(1-\tau)^{\beta-1}(\tau-s)^{\alpha-1}}{p(\tau)} \mathrm{d} \tau, \quad s \in[0,1] .
$$

Remark 2.2 Since $p \in C[0,1]$ and $p(t)>0$ for $t \in[0,1]$, we have $g_{1}(s)>0$ for $s \in[0,1)$ and

$$
\int_{0}^{1} g_{1}(s) \mathrm{d} s=\frac{1}{\Gamma(\alpha+1) \Gamma(\beta)\left(1-m_{1}\right)} \int_{0}^{1} \frac{\tau^{\alpha+1}(1-\tau)^{\beta-1}}{p(\tau)} \mathrm{d} \tau .
$$

Lemma 2.6 (See [11]) The function $K(t, s)$, which is defined by (2.8), has the following properties:

(1) $K(t, s)$ is continuous for any $t, s \in[0,1]$ and $K(t, s)>0$ for any $t, s \in(0,1)$;

(2)

$$
\frac{t^{\beta-1}(1-t) s(1-s)^{\beta-1}}{\Gamma(\beta-1)} \leq K(t, s) \leq \frac{t^{\beta-1}(1-t)(1-s)^{\beta-2}}{\Gamma(\beta)}, \quad t, s \in(0,1)
$$

(3)

$$
K(t, s) \leq \frac{1}{\Gamma(\beta)} t^{\beta-2} s(1-s)^{\beta-1}<\frac{1}{\Gamma(\beta)} t^{\beta-2}, \quad t, s \in(0,1) .
$$


Lemma 2.7 The function $G_{1}(t, s)$, which is defined by (2.7), has the following properties:

(1) $G_{1}(t, s)$ is continuous for $t, s \in[0,1]$ and $G_{1}(t, s)>0$ for $t, s \in(0,1)$;

(2)

$$
\frac{\left(m_{1}-m_{0}\right) s(1-s)^{\beta-1} t^{\beta-1}}{\Gamma(\beta-1)\left(1-m_{1}\right)}<G_{1}(t, s)<\frac{\left(1-m_{1}+m_{2}\right) s(1-s)^{\beta-1} t^{\beta-2}}{\Gamma(\beta)\left(1-m_{1}\right)}, \quad t, s \in(0,1),
$$

where $m_{i}(i=0,1,2)$ are defined by $(2.13)$.

Proof (1) By the expression of $G_{1}(t, s)$ and Lemma 2.6, it is easy to check that (1) holds.

(2) For any $t, s \in(0,1)$, from Lemma 2.6, we have

$$
\begin{aligned}
G_{1}(t, s) & =K(t, s)+\frac{t^{\beta-1}}{1-\int_{0}^{1} s^{\beta-1} \mathrm{~d} A(s)} \int_{0}^{1} K(\tau, s) \mathrm{d} A(\tau) \\
& >\frac{t^{\beta-1}}{1-\int_{0}^{1} s^{\beta-1} \mathrm{~d} A(s)} \int_{0}^{1} \frac{\tau^{\beta-1}(1-\tau) s(1-s)^{\beta-1}}{\Gamma(\beta-1)} \mathrm{d} A(\tau) \\
& =\frac{\left(m_{1}-m_{0}\right) s(1-s)^{\beta-1} t^{\beta-1}}{\Gamma(\beta-1)\left(1-m_{1}\right)} .
\end{aligned}
$$

On the other hand, for any $t, s \in(0,1), 1<\beta \leq 2$, implies that $t^{\beta-1}<t^{\beta-2}$, thus, we have

$$
\begin{aligned}
G_{1}(t, s) & \leq \frac{t^{\beta-2} s(1-s)^{\beta-1}}{\Gamma(\beta)}+\frac{t^{\beta-1}}{1-\int_{0}^{1} s^{\beta-1} \mathrm{~d} A(s)} \int_{0}^{1} \frac{\tau^{\beta-2} s(1-s)^{\beta-1}}{\Gamma(\beta)} \mathrm{d} A(\tau) \\
& <\frac{t^{\beta-2} s(1-s)^{\beta-1}}{\Gamma(\beta)}+\frac{t^{\beta-2} s(1-s)^{\beta-1}}{\Gamma(\beta)\left(1-m_{1}\right)} \int_{0}^{1} \tau^{\beta-2} \mathrm{~d} A(\tau) \\
& =\frac{\left(1-m_{1}+m_{2}\right) s(1-s)^{\beta-1} t^{\beta-2}}{\Gamma(\beta)\left(1-m_{1}\right)} .
\end{aligned}
$$

Lemma 2.8 The function $G(t, s)$, which is defined by (2.11), has the following properties:

(1) $G(t, s)$ is continuous for $t, s \in[0,1]$ and $G(t, s)>0$ for $t, s \in(0,1)$;

(2)

$$
(\beta-1)\left(m_{1}-m_{0}\right) g_{1}(s) t<t^{2-\beta} G(t, s)<\left(1-m_{1}+m_{2}\right) g_{1}(s), \quad t, s \in(0,1),
$$

where $m_{i}, g_{1}(s)$ are defined by (2.13) and (2.15), respectively.

Proof (1) By the expression of $G(t, s)$, we can easily get the results.

(2) According to the definition of $G(t, s)$ and Lemma 2.7, for any $t, s \in(0,1)$, we can obtain that

$$
\begin{aligned}
t^{2-\beta} G(t, s) & =\frac{t^{2-\beta}}{\Gamma(\alpha)} \int_{s}^{1} \frac{1}{p(\tau)} G_{1}(t, \tau)(\tau-s)^{\alpha-1} \mathrm{~d} \tau \\
& >\frac{t^{2-\beta}}{\Gamma(\alpha)} \int_{s}^{1} \frac{\left(m_{1}-m_{0}\right) \tau(1-\tau)^{\beta-1} t^{\beta-1}(\tau-s)^{\alpha-1}}{\Gamma(\beta-1)\left(1-m_{1}\right) p(\tau)} \mathrm{d} \tau \\
& =(\beta-1)\left(m_{1}-m_{0}\right) g_{1}(s) t .
\end{aligned}
$$


On the other hand, for any $t, s \in(0,1)$, by Lemma 2.7 , we can show that

$$
\begin{aligned}
t^{2-\beta} G(t, s) & =\frac{t^{2-\beta}}{\Gamma(\alpha)} \int_{s}^{1} \frac{1}{p(\tau)} G_{1}(t, \tau)(\tau-s)^{\alpha-1} \mathrm{~d} \tau \\
& <\frac{t^{2-\beta}}{\Gamma(\alpha)} \int_{s}^{1} \frac{\left(1-m_{1}+m_{2}\right) \tau(1-\tau)^{\beta-1} t^{\beta-2}(\tau-s)^{\alpha-1}}{\Gamma(\beta)\left(1-m_{1}\right) p(\tau)} \mathrm{d} \tau \\
& =\left(1-m_{1}+m_{2}\right) g_{1}(s) .
\end{aligned}
$$

Let

$$
P=\left\{u \in E: t^{2-\beta} u(t) \geq \gamma_{0} t\|u\|, t \in[0,1]\right\} .
$$

Then $P$ is a cone in $E$.

Lemma 2.9 If $u$ is a positive solution of boundary value problem (1.1), then $u \in P$.

Proof If $u$ is a positive solution of boundary value problem (1.1), then from Definition 2.1, we can get that $u(t)>0$ for $t \in(0,1)$ and $u$ satisfies (2.10). It is easy to see $u \in E$.

For any $t \in[0,1]$, by Lemma 2.8 , we have

$$
\begin{aligned}
t^{2-\beta} u(t) & =t^{2-\beta}\left(\lambda \int_{0}^{1} G(t, s) f(s, u(s)) \mathrm{d} s+a g(t)\right) \\
& \geq \lambda(\beta-1)\left(m_{1}-m_{0}\right) t \int_{0}^{1} g_{1}(s) f(s, u(s)) \mathrm{d} s+\frac{a t\left(m_{2}-m_{1}\right)}{1-m_{1}} \\
& \geq \lambda(\beta-1)\left(m_{1}-m_{0}\right) t \int_{0}^{1} g_{1}(s) f(s, u(s)) \mathrm{d} s+\frac{a t\left(m_{1}-m_{0}\right)}{1-m_{1}} \\
& \geq \frac{t(\beta-1)\left(m_{1}-m_{0}\right)\left(\lambda\left(1-m_{1}\right) \int_{0}^{1} g_{1}(s) f(s, u(s)) \mathrm{d} s+a\right)}{1-m_{1}} .
\end{aligned}
$$

On the other hand, we have

$$
\begin{aligned}
\|u\| & =\sup _{t \in[0,1]} t^{2-\beta}\left|\lambda \int_{0}^{1} G(t, s) f(s, u(s)) \mathrm{d} s+a g(t)\right| \\
& \leq \lambda\left(1-m_{1}+m_{2}\right) \int_{0}^{1} g_{1}(s) f(s, u(s)) \mathrm{d} s+\frac{a \max \left\{1-m_{1}, m_{2}-m_{1}\right\}}{1-m_{1}} \\
& \leq \lambda\left(1-m_{1}+m_{2}\right) \int_{0}^{1} g_{1}(s) f(s, u(s)) \mathrm{d} s+\frac{a\left(1-m_{1}+m_{2}\right)}{1-m_{1}} \\
& =\frac{\left(1-m_{1}+m_{2}\right)\left(\lambda\left(1-m_{1}\right) \int_{0}^{1} g_{1}(s) f(s, u(s)) \mathrm{d} s+a\right)}{1-m_{1}} .
\end{aligned}
$$

Then $t^{2-\beta} u(t) \geq \gamma_{0} t\|u\|$, which implies $u \in P$.

We define $T: P \rightarrow E$ by

$$
(T u)(t)=\lambda \int_{0}^{1} G(t, s) f(s, u(s)) \mathrm{d} s+a g(t) .
$$


Lemma 2.10 The operator $T: P \rightarrow P$ is completely continuous.

Proof By Lemma 2.9, we have Tu $\in P$ for $u \in P$, then $T: P \rightarrow P$.

(1) $T$ is a continuous operator.

If $\left\{u_{n}\right\} \subset P, u \in P$, and $\left\|u_{n}-u\right\| \rightarrow 0$ as $n \rightarrow \infty$, there exists a constant $\gamma>0$ such that $\left\|u_{n}\right\| \leq \gamma$ and $\|u\| \leq \gamma$, that is, $\sup _{t \in[0,1]}\left|t^{2-\beta} u_{n}(t)\right| \leq \gamma$ and $\sup _{t \in[0,1]}\left|t^{2-\beta} u(t)\right| \leq \gamma$. Then there exists $\varphi_{\gamma} \in L^{q}[0,1]$, we have

$$
\begin{aligned}
& t^{2-\beta} G(t, s)\left|f\left(s, u_{n}(s)\right)-f(s, u(s))\right| \\
& \quad \leq 2\left(1-m_{1}+m_{2}\right) g_{1}(s) \varphi_{\gamma}(s) \quad \text { for } t \in[0,1] \text { and a.e. } s \in[0,1] .
\end{aligned}
$$

Since $f$ satisfies the $L^{q}$-Carathéodory conditions, for a.e. $t \in[0,1]$, we have

$$
\lim _{n \rightarrow \infty} f\left(t, u_{n}(t)\right)=\lim _{n \rightarrow \infty} f\left(t, t^{\beta-2} t^{2-\beta} u_{n}(t)\right)=f\left(t, t^{\beta-2} t^{2-\beta} u(t)\right)=f(t, u(t)) .
$$

By the Lebesgue dominated convergence theorem, we can get

$$
\begin{aligned}
\lim _{n \rightarrow \infty}\left\|T u_{n}-T u\right\| & \leq \lim _{n \rightarrow \infty} \sup _{t \in[0,1]} \lambda \int_{0}^{1} t^{2-\beta} G(t, s)\left|f\left(s, u_{n}(s)\right)-f(s, u(s))\right| \mathrm{d} s \\
& \leq \lim _{n \rightarrow \infty} \lambda\left(1-m_{1}+m_{2}\right) \int_{0}^{1} g_{1}(s)\left|f\left(s, u_{n}(s)\right)-f(s, u(s))\right| \mathrm{d} s \\
& =\lambda\left(1-m_{1}+m_{2}\right) \int_{0}^{1} \lim _{n \rightarrow \infty} g_{1}(s)\left|f\left(s, u_{n}(s)\right)-f(s, u(s))\right| \mathrm{d} s \\
& =0, \quad n \rightarrow \infty .
\end{aligned}
$$

Hence, $T: P \rightarrow P$ is continuous.

(2) $T$ is relatively compact.

Let $\Omega \subset P$ be any bounded set, then there exists a constant $r>0$ such that $\|u\| \leq r$ for each $u \in \Omega$, that is, $\sup _{t \in[0,1]}\left|t^{2-\beta} u(t)\right| \leq r$. There exists $\varphi_{r} \in L^{q}[0,1]$, for any $u \in \Omega$, we have

$$
|f(t, u(t))|=\left|f\left(t, t^{\beta-2} t^{2-\beta} u(t)\right)\right| \leq \varphi_{r}(t), \quad \text { a.e. } t \in[0,1]
$$

Therefore, by Lemma 2.8, we have

$$
\begin{aligned}
\|T u\| & =\sup _{t \in[0,1]} t^{2-\beta}\left|\lambda \int_{0}^{1} G(t, s) f(s, u(s)) \mathrm{d} s+a g(t)\right| \\
& \leq \lambda\left(1-m_{1}+m_{2}\right)\left\|\varphi_{r}\right\|_{L^{q}} \int_{0}^{1} g_{1}(s) \mathrm{d} s+\frac{a \max \left\{1-m_{1}, m_{2}-m_{1}\right\}}{1-m_{1}},
\end{aligned}
$$

which implies that $T(\Omega)$ is uniformly bounded.

In addition, because $G(t, s)$ is continuous on $[0,1] \times[0,1]$, then it must be uniformly continuous on $[0,1] \times[0,1]$. Thus, for any $\varepsilon>0$, there exists a constant $\delta \in\left(0, \frac{\varepsilon\left(1-m_{1}\right)}{2 a\left|1-m_{2}\right|+1}\right)$ 
such that

$$
\left|t_{1}^{2-\beta} G\left(t_{1}, s_{1}\right)-t_{2}^{2-\beta} G\left(t_{2}, s_{2}\right)\right|<\frac{\varepsilon}{2 \lambda\left\|\varphi_{r}\right\|_{L^{q}}+1}
$$

whenever $\left|t_{1}-t_{2}\right|<\delta$ and $\left|s_{1}-s_{2}\right|<\delta$, where $t_{1}, t_{2}, s_{1}, s_{2} \in[0,1]$.

Then, for any $u \in \Omega$ and $t_{1}, t_{2} \in[0,1]$ with $\left|t_{1}-t_{2}\right|<\delta$, we have

$$
\begin{aligned}
& \left|t_{1}^{2-\beta} T u\left(t_{1}\right)-t_{2}^{2-\beta} T u\left(t_{2}\right)\right| \\
& \quad=\lambda \int_{0}^{1}\left|t_{1}^{2-\beta} G\left(t_{1}, s\right)-t_{2}^{2-\beta} G\left(t_{2}, s\right)\right| f(s, u(s)) \mathrm{d} s+\frac{a\left|1-m_{2}\right|}{1-m_{1}}\left|t_{1}-t_{2}\right| \\
& \quad \leq \frac{\lambda \varepsilon\left\|\varphi_{r}\right\|_{L^{q}}}{2 \lambda\left\|\varphi_{r}\right\|_{L^{q}}+1}+\frac{a\left|1-m_{2}\right| \delta}{1-m_{1}} \\
& \quad<\varepsilon .
\end{aligned}
$$

Thus, we prove that $T(\Omega)$ is equicontinuous.

According to the Arzela-Ascoli theorem, $T$ is relatively compact.

Therefore, $T: P \rightarrow P$ is completely continuous.

Lemma 2.11 (See [36], Lemma 2.3.1) Let $E$ be a Banach space and $P \subseteq E$ be a cone. Assume that $\Omega$ is a bounded open subset of $E$ and $\theta \in \Omega$ and that $T: P \cap \bar{\Omega} \rightarrow P$ is completely continuous. If

$$
T u \neq \tau u \text { for all } u \in P \cap \partial \Omega \text { and } \tau \geq 1,
$$

then the fixed point index $i(T, P \cap \Omega, P)=1$.

Lemma 2.12 (See [36], Corollary 2.3.1) Let $E$ be a Banach space and $P \subseteq E$ be a cone. Assume that $\Omega$ is a bounded open subset of $E$ and that $T: P \cap \bar{\Omega} \rightarrow P$ is completely continuous. If there exists $u_{0} \in P \backslash\{\theta\}$ such that

$$
u-T u \neq \tau u_{0} \quad \text { for all } u \in P \cap \partial \Omega \text { and } \tau \geq 0,
$$

then the fixed point index $i(T, P \cap \Omega, P)=0$.

Corollary 2.1 Let E be a Banach space and $P \subseteq E$ be a cone. Assume that $\Omega$ is a bounded open subset of $E$ and $\theta \in \Omega$ and that $T: P \cap \bar{\Omega} \rightarrow P$ is completely continuous.

(1) If $\|u\|>\|T u\|$ for $u \in P \cap \partial \Omega$, then $i(T, P \cap \Omega, P)=1$;

(2) If $\|u\|<\|T u\|$ for $u \in P \cap \partial \Omega$, then $i(T, P \cap \Omega, P)=0$.

Proof (1) If $\|u\|>\|T u\|$ for $u \in P \cap \partial \Omega$, then we can show that (2.17) holds.

Otherwise, there exist $u^{*} \in P \cap \partial \Omega$ and $\tau^{*} \geq 1$ such that $T u^{*}=\tau^{*} u^{*}$, then

$$
\left\|T u^{*}\right\|=\left\|\tau^{*} u^{*}\right\| \geq\left\|u^{*}\right\|,
$$

which contradicts $\|u\|>\|T u\|$. In view of Lemma 2.11, we can get $i(T, P \cap \Omega, P)=1$.

(2) If $\|u\|<\|T u\|$ for $u \in P \cap \partial \Omega$, we can prove that (2.18) holds. 
In fact, if for any $u \in P \backslash\{\theta\}$ there exist $u^{*} \in P \cap \partial \Omega$ and $\tau^{*} \geq 0$ such that $u^{*}-T u^{*}=\tau^{*} u$, then

$$
u^{*}=T u^{*}+\tau^{*} u \geq T u^{*} .
$$

Thus, $\left\|u^{*}\right\| \geq\left\|T u^{*}\right\|$, in contradiction with $\|u\|<\|T u\|$.

From Lemma 2.12, we can get $i(T, P \cap \Omega, P)=0$.

\section{The existence of at least one positive solution}

For convenience, we denote

$$
\begin{array}{ll}
f^{\infty}=\limsup _{u \rightarrow+\infty} \sup _{t \in[0,1]} \frac{f\left(t, t^{\beta-2} u\right)}{u} ; & f_{\infty}=\liminf _{u \rightarrow+\infty} \inf _{t \in\left[\frac{1}{4}, \frac{3}{4}\right]} \frac{f\left(t, t^{\beta-2} u\right)}{u} ; \\
f^{0}=\limsup _{u \rightarrow 0^{+}} \sup _{t \in[0,1]} \frac{f\left(t, t^{\beta-2} u\right)}{u} ; & f_{0}=\liminf _{u \rightarrow 0^{+}} \inf _{t \in\left[\frac{1}{4}, \frac{3}{4}\right]} \frac{f\left(t, t^{\beta-2} u\right)}{u} .
\end{array}
$$

Let $B_{r}=\{u \in E:\|u\|<r\}, \partial B_{r}=\{u \in E:\|u\|=r\}, P_{r}=P \cap B_{r}, \partial P_{r}=P \cap \partial B_{r}$.

Theorem 3.1 Suppose that there exist constants $\xi, \eta>0$ such that $f^{0}<\xi$ and $f_{\infty}>\eta$. If $\xi<\frac{\gamma_{0}^{2} \eta \int_{\frac{1}{4}}^{\frac{3}{4}} g_{1}(s) \mathrm{d} s}{4 \int_{0}^{1} g_{1}(s) \mathrm{d} s}$ and $\lambda$ satisfies

$$
4\left((\beta-1)\left(m_{1}-m_{0}\right) \gamma_{0} \eta \int_{\frac{1}{4}}^{\frac{3}{4}} g_{1}(s) \mathrm{d} s\right)^{-1} \leq \lambda \leq\left(\left(1-m_{1}+m_{2}\right) \xi \int_{0}^{1} g_{1}(s) \mathrm{d} s\right)^{-1}
$$

then there exists a constant $a_{\lambda}>0$ such that boundary value problem (1.1) with $0 \leq a \leq a_{\lambda}$ has at least one positive solution.

Proof Since $f^{0}<\xi$, there exists a constant $r_{1}>0$ such that

$$
f\left(t, t^{\beta-2} u\right)<\xi u, \quad t \in(0,1] \text {, and } u \in\left[0, r_{1}\right]
$$

Let

$$
a_{\lambda}=\frac{\left(1-m_{1}\right)\left(1-\lambda\left(1-m_{1}+m_{2}\right) \xi \int_{0}^{1} g_{1}(s) \mathrm{d} s\right) r_{1}}{\max \left\{1-m_{1}, m_{2}-m_{1}\right\}} .
$$

Because $\lambda$ satisfies (3.1) and $0 \leq a \leq a_{\lambda}$, by Lemma 2.8, for any $u \in \partial P_{r_{1}}$, we have $0<$ $t^{2-\beta} u(t) \leq r_{1}$ for $t \in(0,1]$ and

$$
\begin{aligned}
\|T u\| & =\sup _{t \in[0,1]} t^{2-\beta}\left|\lambda \int_{0}^{1} G(t, s) f(s, u(s)) \mathrm{d} s+\operatorname{ag}(t)\right| \\
& <\lambda\left(1-m_{1}+m_{2}\right) \xi \int_{0}^{1} g_{1}(s) s^{2-\beta} u(s) \mathrm{d} s+\frac{a_{\lambda} \max \left\{1-m_{1}, m_{2}-m_{1}\right\}}{1-m_{1}} \\
& \leq \lambda\left(1-m_{1}+m_{2}\right) \xi r_{1} \int_{0}^{1} g_{1}(s) \mathrm{d} s+\frac{a_{\lambda} \max \left\{1-m_{1}, m_{2}-m_{1}\right\}}{1-m_{1}} \\
& =r_{1} .
\end{aligned}
$$


Hence,

$$
\|T u\|<\|u\|, \quad u \in \partial P_{r_{1}} .
$$

It follows from Corollary 2.1(1) $i\left(T, P_{r_{1}}, P\right)=1$.

By $f_{\infty}>\eta$, there exists a constant $r_{2}>r_{1}$ such that

$$
f\left(t, t^{\beta-2} u\right)>\eta u, \quad t \in\left[\frac{1}{4}, \frac{3}{4}\right] \text { and } u \in\left[\frac{1}{4} \gamma_{0} r_{2},+\infty\right) \text {. }
$$

For any $u \in \partial P_{r_{2}}$, we have

$$
t^{2-\beta} u(t) \geq \gamma_{0} t\|u\| \geq \frac{1}{4} \gamma_{0} r_{2}, \quad t \in\left[\frac{1}{4}, \frac{3}{4}\right]
$$

and by Lemma 2.8,

$$
\begin{aligned}
\|T u\| & =\sup _{t \in[0,1]} t^{2-\beta}\left|\lambda \int_{0}^{1} G(t, s) f(s, u(s)) \mathrm{d} s+a g(t)\right| \\
& >\sup _{t \in[0,1]} \lambda(\beta-1)\left(m_{1}-m_{0}\right) t \int_{\frac{1}{4}}^{\frac{3}{4}} g_{1}(s) f\left(s, s^{\beta-2} s^{2-\beta} u(s)\right) \mathrm{d} s \\
& >\lambda(\beta-1)\left(m_{1}-m_{0}\right) \int_{\frac{1}{4}}^{\frac{3}{4}} \eta s^{2-\beta} u(s) g_{1}(s) \mathrm{d} s \\
& \geq \frac{1}{4} \lambda(\beta-1)\left(m_{1}-m_{0}\right) \gamma_{0} r_{2} \eta \int_{\frac{1}{4}}^{\frac{3}{4}} g_{1}(s) \mathrm{d} s \\
& \geq r_{2} .
\end{aligned}
$$

Therefore,

$$
\|T u\|>\|u\|, \quad u \in \partial P_{r_{2}} .
$$

It follows from Corollary 2.1(2) $i\left(T, P_{r_{2}}, P\right)=0$.

According to the additivity property of the fixed point index, we obtain

$$
i\left(T, P_{r_{2}} \backslash \bar{P}_{r_{1}}, P\right)=i\left(T, P_{r_{2}}, P\right)-i\left(T, P_{r_{1}}, P\right)=-1
$$

Then $T$ has at least one fixed point $u \in P \cap\left(P_{r_{2}} \backslash \bar{P}_{r_{1}}\right)$ with $r_{1}<\|u\|<r_{2}$. Because $u \in P$, we have $t^{2-\beta} u(t) \geq \gamma_{0} t\|u\|>0$ for $t \in(0,1]$, that is, $u(t)>0$ for $t \in(0,1), u=u(t)$ is a positive solution for boundary value problem (1.1) with $0 \leq a \leq a_{\lambda}$.

Theorem 3.2 If $f^{0}=0, f_{\infty}=+\infty$, and $\lambda>0$, then there exists a constant $a_{\lambda}>0$ such that boundary value problem (1.1) with $0 \leq a \leq a_{\lambda}$ has at least one positive solution.

Proof Let $\lambda>0,0<\xi<\left(\lambda\left(1-m_{1}+m_{2}\right) \int_{0}^{1} g_{1}(s) \mathrm{d} s\right)^{-1}$ and $\eta \geq 4\left(\lambda(\beta-1)\left(m_{1}-m_{0}\right) \gamma_{0} \times\right.$ $\left.\int_{\frac{1}{4}}^{\frac{3}{4}} g_{1}(s) \mathrm{d} s\right)^{-1}$. 
By $f^{0}=0$, there exists a constant $r_{1}>0$ such that

$$
f\left(t, t^{\beta-2} u\right)<\xi u, \quad t \in(0,1], \text { and } u \in\left[0, r_{1}\right],
$$

and by $f_{\infty}=+\infty$, there exists a constant $r_{2}>r_{1}$ such that

$$
f\left(t, t^{\beta-2} u\right)>\eta u, \quad t \in\left[\frac{1}{4}, \frac{3}{4}\right] \text { and } u \in\left[\frac{1}{4} \gamma_{0} r_{2},+\infty\right) .
$$

Let

$$
\boldsymbol{a}_{\lambda}=\frac{\left(1-m_{1}\right)\left(1-\lambda\left(1-m_{1}+m_{2}\right) \xi \int_{0}^{1} g_{1}(s) \mathrm{d} s\right) r_{1}}{\max \left\{1-m_{1}, m_{2}-m_{1}\right\}} .
$$

For $0 \leq a \leq a_{\lambda}$, similar to the proof of Theorem 3.1, we have

$$
i\left(T, P_{r_{1}}, P\right)=1
$$

and

$$
i\left(T, P_{r_{2}}, P\right)=0 .
$$

According to the additivity property of the fixed point index,

$$
i\left(T, P_{r_{2}} \backslash \bar{P}_{r_{1}}, P\right)=i\left(T, P_{r_{2}}, P\right)-i\left(T, P_{r_{1}}, P\right)=-1 .
$$

Then $T$ has at least one fixed point $u \in P \cap\left(P_{r_{2}} \backslash \bar{P}_{r_{1}}\right)$ with $r_{1}<\|u\|<r_{2}$, that is, $u$ is a positive solution for boundary value problem (1.1) with $0 \leq a \leq a_{\lambda}$.

Theorem 3.3 Suppose that there exist constants $\xi, \eta>0$ such that $f^{\infty}<\xi$ and $f_{0}>\eta$. If $\xi<\frac{\gamma_{0}^{2} \eta \int_{\frac{1}{4}}^{\frac{3}{4}} g_{1}(s) \mathrm{d} s}{12 \int_{0}^{1} g_{1}(s) \mathrm{d} s}$ and $\lambda$ satisfies

$$
4\left((\beta-1)\left(m_{1}-m_{0}\right) \gamma_{0} \eta \int_{\frac{1}{4}}^{\frac{3}{4}} g_{1}(s) \mathrm{d} s\right)^{-1} \leq \lambda \leq\left(3\left(1-m_{1}+m_{2}\right) \xi \int_{0}^{1} g_{1}(s) \mathrm{d} s\right)^{-1},
$$

then boundary value problem (1.1) with $a \geq 0$ has at least one positive solution.

Proof By $f_{0}>\eta$, there exists a constant $R_{1}>0$ such that

$$
f\left(t, t^{\beta-2} u\right)>\eta u, \quad t \in\left[\frac{1}{4}, \frac{3}{4}\right] \text {, and } u \in\left[0, R_{1}\right] .
$$

When $\lambda$ satisfies (3.3) and $a \geq 0$, similar to the proof of Theorem 3.1, we can obtain

$$
\|T u\|>\|u\|, \quad u \in \partial P_{R_{1}},
$$

and

$$
i\left(T, P_{R_{1}}, P\right)=0 .
$$


On the other hand, by $f^{\infty}<\xi$, there exists a constant $M>0$ such that

$$
f\left(t, t^{\beta-2} u\right)<\xi u, \quad t \in[0,1], \text { and } u \in[M,+\infty)
$$

Since $f$ satisfies the $L^{q}$-Carathéodory conditions, for the above $M>0$, there exists $\varphi_{M} \in$ $L^{q}[0,1]$ such that

$$
\left|f\left(t, t^{\beta-2} u\right)\right| \leq \varphi_{M}(t), \quad \text { a.e. } t \in[0,1] \text { and } u \in[0, M] \text {. }
$$

Let

$$
R_{2}>\max \left\{M, R_{1}, 3 \lambda\left(1-m_{1}+m_{2}\right) \int_{0}^{1} g_{1}(s) \varphi_{M}(s) \mathrm{d} s, \frac{3 a \max \left\{1-m_{1}, m_{2}-m_{1}\right\}}{1-m_{1}}\right\} .
$$

For any $u \in \partial P_{R_{2}}$, by Lemma 2.8 , we have

$$
\begin{aligned}
\|T u\|= & \sup _{t \in[0,1]} t^{2-\beta}\left|\lambda \int_{0}^{1} G(t, s) f(s, u(s)) \mathrm{d} s+a g(t)\right| \\
\leq & \lambda\left(1-m_{1}+m_{2}\right)\left(\int_{0 \leq s^{2-\beta} u(s) \leq M} g_{1}(s) f(s, u(s)) \mathrm{d} s+\int_{s^{2-\beta} u(s) \geq M} g_{1}(s) f(s, u(s)) \mathrm{d} s\right) \\
& +\frac{a \max \left\{1-m_{1}, m_{2}-m_{1}\right\}}{1-m_{1}} \\
< & \lambda\left(1-m_{1}+m_{2}\right)\left(\int_{0}^{1} g_{1}(s) \varphi_{M}(s) \mathrm{d} s+\xi R_{2} \int_{0}^{1} g_{1}(s) \mathrm{d} s\right) \\
& +\frac{a \max \left\{1-m_{1}, m_{2}-m_{1}\right\}}{1-m_{1}} \\
< & \frac{R_{2}}{3}+\frac{R_{2}}{3}+\frac{R_{2}}{3} \\
= & R_{2} .
\end{aligned}
$$

That is,

$$
\|T u\|<\|u\|, \quad u \in \partial P_{R_{2}} .
$$

From Corollary 2.1(1), we can get $i\left(T, P_{R_{2}}, P\right)=1$.

According to the additivity property of the fixed point index, we obtain

$$
i\left(T, P_{R_{2}} \backslash \bar{P}_{R_{1}}, P\right)=i\left(T, P_{R_{2}}, P\right)-i\left(T, P_{R_{1}}, P\right)=1 \text {. }
$$

Then $T$ has at least one fixed point $u \in P \cap\left(P_{R_{2}} \backslash \bar{P}_{R_{1}}\right)$ with $R_{1}<\|u\|<R_{2}$. That is, $u$ is a positive solution for boundary value problem (1.1) with $a \geq 0$.

Theorem 3.4 If $f_{0}=+\infty, f^{\infty}=0, \lambda>0$, and $a \geq 0$, then boundary value problem (1.1) has at least one positive solution. 
Proof Denote

$$
\begin{aligned}
& \eta=4\left(\lambda(\beta-1)\left(m_{1}-m_{0}\right) \gamma_{0} \int_{\frac{1}{4}}^{\frac{3}{4}} g_{1}(s) \mathrm{d} s\right)^{-1}, \\
& \xi=\left(3 \lambda\left(1-m_{1}+m_{2}\right) \int_{0}^{1} g_{1}(s) \mathrm{d} s\right)^{-1} .
\end{aligned}
$$

By $f_{0}=+\infty$, there exists a constant $R_{1}>0$ such that

$$
f\left(t, t^{\beta-2} u\right) \geq \eta u, \quad t \in\left[\frac{1}{4}, \frac{3}{4}\right] \text {, and } u \in\left[0, R_{1}\right]
$$

On the other hand, by $f^{\infty}=0$, there exists a constant $M>0$ such that

$$
f\left(t, t^{\beta-2} u\right) \leq \xi u, \quad t \in(0,1] \text {, and } u \in[M,+\infty) .
$$

Let

$$
R_{2}>\max \left\{M, R_{1}, 3 \lambda\left(1-m_{1}+m_{2}\right) \int_{0}^{1} g_{1}(s) \varphi_{M}(s) \mathrm{d} s, \frac{3 a \max \left\{1-m_{1}, m_{2}-m_{1}\right\}}{1-m_{1}}\right\} .
$$

Similar to the proof of Theorem 3.3, we can obtain $T$ has at least one fixed point $u \in$ $P \cap\left(P_{R_{2}} \backslash \bar{P}_{R_{1}}\right)$ with $R_{1}<\|u\|<R_{2}$. That is, $u=u(t)$ is a positive solution for boundary value problem (1.1).

Theorem 3.5 If $f_{\infty}=+\infty, r>0$ is a constant and $\lambda$ satisfies

$$
0<\lambda \leq r\left(\left(1-m_{1}+m_{2}\right) \int_{0}^{1} g_{1}(s) \varphi_{r}(s) \mathrm{d} s\right)^{-1}
$$

then there exists a constant $a_{\lambda}>0$ such that boundary value problem (1.1) with $0 \leq a \leq a_{\lambda}$ has at least one positive solution $u$ with $\|u\|>r$.

Proof For any given $r>0$, when $\lambda$ satisfies (3.4), let

$$
a_{\lambda}=\frac{\left(1-m_{1}\right)\left(r-\lambda\left(1-m_{1}+m_{2}\right) \int_{0}^{1} g_{1}(s) \varphi_{r}(s) \mathrm{d} s\right)}{\max \left\{1-m_{1}, m_{2}-m_{1}\right\}} .
$$

For $0 \leq a \leq a_{\lambda}$ and any $u \in \partial P_{r}$, by Lemma 2.8, we have

$$
\begin{aligned}
\|T u\| & =\sup _{t \in[0,1]} t^{2-\beta}\left|\lambda \int_{0}^{1} G(t, s) f(s, u(s)) \mathrm{d} s+\operatorname{ag}(t)\right| \\
& <\lambda\left(1-m_{1}+m_{2}\right) \int_{0}^{1} g_{1}(s) \varphi_{r}(s) \mathrm{d} s+\frac{a_{\lambda} \max \left\{1-m_{1}, m_{2}-m_{1}\right\}}{1-m_{1}} \\
& =r .
\end{aligned}
$$

That is,

$$
\|T u\|<\|u\|, \quad u \in \partial P_{r} .
$$


By Corollary 2.1(1), we can get $i\left(T, P_{r}, P\right)=1$.

Let $\eta=4\left(\lambda(\beta-1)\left(m_{1}-m_{0}\right) \gamma_{0} \int_{\frac{1}{4}}^{\frac{3}{4}} g_{1}(s) \mathrm{d} s\right)^{-1}$. Since $f_{\infty}=+\infty$, there exists a constant $r_{1}>r$ such that

$$
f\left(t, t^{\beta-2} u\right) \geq \eta u, \quad t \in\left[\frac{1}{4}, \frac{3}{4}\right] \text {, and } u \in\left[\frac{1}{4} \gamma_{0} r_{1},+\infty\right) .
$$

Similar to the proof of Theorem 3.2, we obtain

$$
\|T u\|>\|u\|, \quad u \in \partial P_{r_{1}} .
$$

By Corollary 2.1(2)

$$
i\left(T, P_{r_{1}}, P\right)=0 .
$$

According to the additivity property of the fixed point index,

$$
i\left(T, P_{r_{1}} \backslash \bar{P}_{r}, P\right)=i\left(T, P_{r_{1}}, P\right)-i\left(T, P_{r}, P\right)=-1 .
$$

Then $T$ has at least one fixed point $u \in P \cap\left(P_{r_{1}} \backslash \bar{P}_{r}\right)$ with $r<\|u\|<r_{1}$. That is, $u=u(t)$ is a positive solution for boundary value problem (1.1) with $0 \leq a \leq a_{\lambda}$.

\section{The multiplicity and nonexistence of positive solutions}

In this section, we present the existence of at least two positive solutions and nonexistence positive solutions.

Theorem 4.1 Suppose that there exist constants $\eta_{1}, \eta_{2}>0$ such that $f_{0}>\eta_{1}$ and $f_{\infty}>\eta_{2}$. Let a constant

$$
r>4 \int_{0}^{1} g_{1}(s) \varphi_{r}(s) \mathrm{d} s\left(\min \left\{\eta_{1}, \eta_{2}\right\} \gamma_{0}^{2} \int_{\frac{1}{4}}^{\frac{3}{4}} g_{1}(s) \mathrm{d} s\right)^{-1} .
$$

If $\lambda$ satisfies

$$
\begin{aligned}
& 4\left(\min \left\{\eta_{1}, \eta_{2}\right\}(\beta-1)\left(m_{1}-m_{0}\right) \gamma_{0} \int_{\frac{1}{4}}^{\frac{3}{4}} g_{1}(s) \mathrm{d} s\right)^{-1} \\
& \leq \lambda \leq r\left(\left(1-m_{1}+m_{2}\right) \int_{0}^{1} g_{1}(s) \varphi_{r}(s) \mathrm{d} s\right)^{-1}
\end{aligned}
$$

then there exists a constant $a_{\lambda} \geq 0$ such that boundary value problem (1.1) with $0 \leq a \leq a_{\lambda}$ has at least two positive solutions $u_{1}$ and $u_{2}$.

Proof Let

$$
a_{\lambda}=\frac{\left(1-m_{1}\right)\left(r-\lambda\left(1-m_{1}+m_{2}\right) \int_{0}^{1} g_{1}(s) \varphi_{r}(s) \mathrm{d} s\right)}{\max \left\{1-m_{1}, m_{2}-m_{1}\right\}},
$$

by (4.1) and (4.2), we have $a_{\lambda} \geq 0$. 
For $0 \leq a \leq a_{\lambda}$ and any $u \in \partial P_{r}$, similar to the proof of Theorem 3.5, we have

$$
\|T u\|<\|u\|, \quad u \in \partial P_{r},
$$

and

$$
i\left(T, P_{r}, P\right)=1 \text {. }
$$

Since $f_{0}>\eta_{1}$, there exists a constant $0<\bar{r}_{1}<r$ such that

$$
f\left(t, t^{\beta-2} u\right)>\eta_{1} u, \quad t \in\left[\frac{1}{4}, \frac{3}{4}\right] \text {, and } u \in\left[0, \bar{r}_{1}\right]
$$

By $f_{\infty}>\eta_{2}$, there exists a constant $\bar{r}_{2}>r$ such that

$$
f\left(t, t^{\beta-2} u\right)>\eta_{2} u, \quad t \in\left[\frac{1}{4}, \frac{3}{4}\right] \text {, and } u \in\left[\frac{1}{4} \gamma_{0} \bar{r}_{2},+\infty\right) .
$$

Similar to the proof of Theorem 3.3 and Theorem 3.1, we can obtain $\|T u\|>\|u\|, u \in$ $\partial P_{\bar{r}_{1}}$, and $\|T u\|>\|u\|, u \in \partial P_{\bar{r}_{2}}$. Hence, by Corollary 2.1(2), we can get $i\left(T, P_{\bar{r}_{1}}, P\right)=0$ and $i\left(T, P_{\bar{r}_{2}}, P\right)=0$.

According to the additivity property of the fixed point index, we can show

$$
i\left(T, P_{r} \backslash \bar{P}_{\bar{r}_{1}}, P\right)=i\left(T, P_{r}, P\right)-i\left(T, P_{\bar{r}_{1}}, P\right)=1
$$

and

$$
i\left(T, P_{\bar{r}_{2}} \backslash \bar{P}_{r}, P\right)=i\left(T, P_{\bar{r}_{2}}, P\right)-i\left(T, P_{r}, P\right)=-1 .
$$

Then $T$ has at least two fixed points $u_{1} \in P \cap\left(P_{r} \backslash \bar{P}_{\bar{r}_{1}}\right)$ with $\bar{r}_{1}<\left\|u_{1}\right\|<r$ and $u_{2} \in P \cap$ $\left(\bar{P}_{\bar{r}_{2}} \backslash P_{r}\right)$ with $r<\left\|u_{2}\right\|<\bar{r}_{2}$. That is, $u_{1}$ and $u_{2}$ are positive solutions of boundary value problem (1.1) with $0 \leq a \leq a_{\lambda}$.

Theorem 4.2 Suppose that $f_{0}=+\infty, f_{\infty}=+\infty$, and a constant $r>0$ hold. If $\lambda$ satisfies

$$
0<\lambda \leq r\left(\left(1-m_{1}+m_{2}\right) \int_{0}^{1} g_{1}(s) \varphi_{r}(s) \mathrm{d} s\right)^{-1}
$$

then there exists a constant $a_{\lambda} \geq 0$ such that boundary value problem (1.1) with $0 \leq a \leq a_{\lambda}$ has at least two positive solutions $u_{1}$ and $u_{2}$.

Proof Let

$$
\boldsymbol{a}_{\lambda}=\frac{\left(1-m_{1}\right)\left(r-\lambda\left(1-m_{1}+m_{2}\right) \int_{0}^{1} g_{1}(s) \varphi_{r}(s) \mathrm{d} s\right)}{\max \left\{1-m_{1}, m_{2}-m_{1}\right\}} .
$$

Since $\lambda$ satisfies (4.3), we have $a_{\lambda} \geq 0$. 
For $0 \leq a \leq a_{\lambda}$ and any $u \in \partial P_{r}$, similar to the proof of Theorem 3.5, we have

$$
\|T u\|<\|u\| \quad \text { for } u \in \partial P_{r} .
$$

By Corollary 2.1(1), we can get $i\left(T, P_{r}, P\right)=1$.

Denote

$$
\eta_{1}=\eta_{2}=4\left(\lambda(\beta-1)\left(m_{1}-m_{0}\right) \gamma_{0} \int_{\frac{1}{4}}^{\frac{3}{4}} g_{1}(s) \mathrm{d} s\right)^{-1} .
$$

By $f_{0}=+\infty$, there exists a constant $0<\bar{r}_{1}<r$ such that

$$
f\left(t, t^{\beta-2} u\right) \geq \eta_{1} u, \quad t \in\left[\frac{1}{4}, \frac{3}{4}\right], \text { and } u \in\left[0, \bar{r}_{1}\right] .
$$

And by $f_{\infty}=+\infty$, there exists a constant $\bar{r}_{2}>r$ such that

$$
f\left(t, t^{\beta-2} u\right) \geq \eta_{2} u, \quad t \in\left[\frac{1}{4}, \frac{3}{4}\right] \text {, and } u \in\left[\frac{1}{4} \gamma_{0} \bar{r}_{2},+\infty\right) .
$$

Similar to the proof of Theorem 3.3 and Theorem 3.1, we obtain

$$
\|T u\|>\|u\|, \quad u \in \partial P_{\bar{r}_{1}}, \quad \text { and } \quad\|T u\|>\|u\|, \quad u \in \partial P_{\bar{r}_{2}} .
$$

By Corollary 2.1(2), we can get $i\left(T, P_{\bar{r}_{1}}, P\right)=0$ and $i\left(T, P_{\bar{r}_{2}}, P\right)=0$.

According to the additivity property of the fixed point index, we obtain

$$
i\left(T, P_{r} \backslash \bar{P}_{\bar{r}_{1}}, P\right)=i\left(T, P_{r}, P\right)-i\left(T, P_{\bar{r}_{1}}, P\right)=1
$$

and

$$
i\left(T, P_{\bar{r}_{2}} \backslash \bar{P}_{r}, P\right)=i\left(T, P_{\bar{r}_{2}}, P\right)-i\left(T, P_{r}, P\right)=-1 .
$$

Then $T$ has at least two fixed points $u_{1} \in P \cap\left(P_{r} \backslash \bar{P}_{\bar{r}_{1}}\right)$ with $\bar{r}_{1}<\left\|u_{1}\right\|<r$, and $u_{2} \in P \cap$ $\left(\bar{P}_{\bar{r}_{2}} \backslash P_{r}\right)$ with $r<\left\|u_{2}\right\|<\bar{r}_{2}$. That is, $u_{1}$ and $u_{2}$ are positive solutions for boundary value problem (1.1) with $0 \leq a \leq a_{\lambda}$.

Theorem 4.3 Suppose that $0<\liminf _{u \rightarrow+\infty} \inf _{t \in\left[\frac{1}{4}, \frac{3}{4}\right]} f\left(t, t^{\beta-2} u\right)<+\infty, f^{0}=0$, and $f^{\infty}=0$ hold. Then there exist constants $\lambda^{*}>0$ and $a_{0}>0$ such that boundary value problem (1.1) has at least two positive solutions with $\lambda \geq \lambda^{*}$ and $0 \leq a \leq a_{0}$.

Proof By $0<\liminf _{u \rightarrow+\infty} \inf _{t \in\left[\frac{1}{4}, \frac{3}{4}\right]} f\left(t, t^{\beta-2} u\right)<+\infty$, there exist constants $L>0$ and $R_{2}>0$ such that

$$
f\left(t, t^{\beta-2} u\right)>L, \quad t \in\left[\frac{1}{4}, \frac{3}{4}\right] \text {, and } u \in\left[R_{2},+\infty\right),
$$

Let

$$
\lambda^{*}=\frac{4 R_{2}}{\left(m_{1}-m_{0}\right)(\beta-1) L \int_{\frac{1}{4}}^{\frac{3}{4}} g_{1}(s) \mathrm{d} s} .
$$


We denote $\left.\xi=\left(3 \lambda\left(1-m_{1}+m_{2}\right) \int_{0}^{1} g_{1}(s) \mathrm{d} s\right)\right)^{-1}$ for $\lambda \geq \lambda^{*}$.

By $f^{\infty}=0$, there exists a constant $M>R_{2}$ such that

$$
f\left(t, t^{\beta-2} u\right) \leq \xi u, \quad t \in(0,1] \text {, and } u \in[M,+\infty) .
$$

By $f^{0}=0$, there exists a constant $0<R_{1}<R_{2}$ such that

$$
f\left(t, t^{\beta-2} u\right) \leq \xi u, \quad t \in(0,1] \text {, and } u \in\left[0, R_{1}\right] .
$$

Let

$$
R_{3}>\left\{4^{2-\beta} R_{2}, 3 \lambda\left(1-m_{1}+m_{2}\right) \int_{0}^{1} g_{1}(s) \varphi_{M}(s) \mathrm{d} s\right\},
$$

for $0<R_{1}<R_{2}<R_{3}$, we define

$$
\begin{aligned}
& \Omega_{1}=\left\{u: u \in E,\|u\|<R_{1}\right\}, \\
& \Omega_{2}=\left\{u: u \in E,\|u\|<R_{3}, \min _{t \in\left[\frac{1}{4}, \frac{3}{4}\right]} t^{2-\beta} u(t)>R_{2}\right\}, \\
& \Omega_{3}=\left\{u: u \in E,\|u\|<R_{3}\right\} .
\end{aligned}
$$

It is easy to see that $\Omega_{1}, \Omega_{2}$, and $\Omega_{3}$ are nonempty bounded convex open sets in $E$, and $\Omega_{1} \subset \Omega_{3}, \Omega_{2} \subset \Omega_{3}$, and $\Omega_{1} \cap \Omega_{2}=\varnothing$. Let

$$
a_{0}=\frac{R_{1}\left(1-m_{1}\right)}{3 \max \left\{1-m_{1}, m_{2}-m_{1}\right\}} .
$$

Then, when $0 \leq a \leq a_{0}$, for any $u \in P \cap \partial \Omega_{1}$, similar to Theorem 3.1, we obtain

$$
\|T u\|<\|u\|, \quad u \in P \cap \partial \Omega_{1}
$$

and by Corollary 2.1(1), we can get that

$$
i\left(T, P \cap \Omega_{1}, P\right)=1 \text {. }
$$

For any $u \in P \cap \partial \Omega_{3}$, by Lemma 2.8 , we have

$$
\begin{aligned}
& \|T u\|=\sup _{t \in[0,1]} t^{2-\beta}\left|\lambda \int_{0}^{1} G(t, s) f(s, u(s)) \mathrm{d} s+a g(t)\right|
\end{aligned}
$$

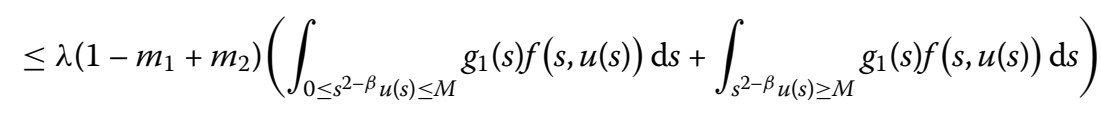

$$
\begin{aligned}
& +\frac{a \max \left\{1-m_{1}, m_{2}-m_{1}\right\}}{1-m_{1}} \\
& <\lambda\left(1-m_{1}+m_{2}\right)\left(\int_{0}^{1} g_{1}(s) \varphi_{M}(s) \mathrm{d} s+\xi R_{3} \int_{0}^{1} g_{1}(s) \mathrm{d} s\right)
\end{aligned}
$$




$$
\begin{aligned}
& +\frac{a \max \left\{1-m_{1}, m_{2}-m_{1}\right\}}{1-m_{1}} \\
& <\frac{R_{3}}{3}+\frac{R_{3}}{3}+\frac{R_{3}}{3}=R_{3},
\end{aligned}
$$

that is,

$$
\|T u\|<\|u\| \quad \text { for } u \in P \cap \partial \Omega_{3},
$$

it follows $i\left(T, P \cap \Omega_{3}, P\right)=1$ from Corollary 2.1(1).

Similarly, for any $u \in P \cap \partial \Omega_{2}$, we have

$$
\begin{aligned}
\|T u\| \leq & \lambda\left(1-m_{1}+m_{2}\right)\left(\int_{0 \leq s^{2-\beta} u(s) \leq M} g_{1}(s) f(s, u(s)) \mathrm{d} s+\int_{s^{2-\beta} u(s) \geq M} g_{1}(s) f(s, u(s)) \mathrm{d} s\right) \\
& +\frac{a \max \left\{1-m_{1}, m_{2}-m_{1}\right\}}{1-m_{1}} \\
< & \lambda\left(1-m_{1}+m_{2}\right)\left(\int_{0}^{1} g_{1}(s) \varphi_{M}(s) \mathrm{d} s+\xi R_{3} \int_{0}^{1} g_{1}(s) \mathrm{d} s\right) \\
& +\frac{a \max \left\{1-m_{1}, m_{2}-m_{1}\right\}}{1-m_{1}} \\
< & \frac{R_{3}}{3}+\frac{R_{3}}{3}+\frac{R_{3}}{3} \\
= & R_{3},
\end{aligned}
$$

then $\|T u\|<R_{3}$, and

$$
\begin{aligned}
\min _{t \in\left[\frac{1}{4}, \frac{3}{4}\right]} t^{2-\beta} T u(t) & \geq \min _{t \in\left[\frac{1}{4}, \frac{3}{4}\right]} t^{2-\beta} \lambda \int_{0}^{1} G(t, s) f(s, u(s)) \mathrm{d} s \\
& \geq \min _{t \in\left[\frac{1}{4}, \frac{3}{4}\right]} \lambda(\beta-1)\left(m_{1}-m_{0}\right) t \int_{\frac{1}{4}}^{\frac{3}{4}} g_{1}(s) f(s, u(s)) \mathrm{d} s \\
& >\frac{1}{4} \lambda^{*}(\beta-1)\left(m_{1}-m_{0}\right) L \int_{\frac{1}{4}}^{\frac{3}{4}} g_{1}(s) \mathrm{d} s \\
& =R_{2},
\end{aligned}
$$

so, $T u \in P \cap \Omega_{2}$.

Let $u_{0} \equiv \frac{1}{2}\left(4^{2-\beta} R_{2}+R_{3}\right)$, and

$$
H(\tau, u)=(1-\tau) T u+\tau u_{0}, \quad(\tau, u) \in[0,1] \times\left(P \cap \bar{\Omega}_{2}\right) .
$$

Because $u_{0} \in E,\left\|u_{0}\right\|=\frac{1}{2}\left(4^{2-\beta} R_{2}+R_{3}\right), \gamma_{0}<1$, then $t^{2-\beta} u_{0} \geq \gamma_{0} t\left\|u_{0}\right\|$, that is, $u_{0} \in P$. Since $R_{3}>4^{2-\beta} R_{2}$, we can see that $\left\|u_{0}\right\|<R_{3}, \min _{t \in\left[\frac{1}{4}, \frac{3}{4}\right]} t^{2-\beta} u_{0}>R_{2}$, which implies $u_{0} \in$ $P \cap \Omega_{2}$. So, we have

$$
H(\tau, u) \in P \cap \Omega_{2}, \quad(\tau, u) \in[0,1] \times\left(P \cap \bar{\Omega}_{2}\right) .
$$


Therefore, we have

$$
H(\tau, u) \neq u, \quad(\tau, u) \in[0,1] \times\left(P \cap \partial \Omega_{2}\right) .
$$

By the complete continuity of the operator $T$ and the definition of $H$, we can know that $H:[0,1] \times\left(P \cap \Omega_{2}\right) \rightarrow P$ is completely continuous.

According to the homotopy invariance and normality of fixed point index,

$$
i\left(T, P \cap \Omega_{2}, P\right)=i\left(u_{0}, P \cap \Omega_{2}, P\right)=1 \text {. }
$$

Thus, $T$ has one fixed point $u_{1}$ in $P \cap \Omega_{2}$.

By the additivity of the fixed point index, we obtain

$$
\begin{aligned}
i( & \left.T, P \cap\left(\Omega_{3} \backslash\left(\bar{\Omega}_{1} \cup \bar{\Omega}_{2}\right)\right), P\right) \\
& =i\left(T, P \cap \Omega_{3}, P\right)-i\left(T, P \cap \Omega_{2}, P\right)-i\left(T, P \cap \Omega_{1}, P\right) \\
& =1-1-1=-1 .
\end{aligned}
$$

Thus, $T$ has one fixed point $u_{2}$ in $P \cap\left(\Omega_{3} \backslash\left(\bar{\Omega}_{1} \cup \bar{\Omega}_{2}\right)\right)$.

Consequently, $u_{1}$ and $u_{2}$ are positive solutions of boundary value problem (1.1) with $\lambda \geq \lambda^{*}$ and $0 \leq a \leq a_{0}$.

Remark 4.1 If $f(t, u) \neq 0$, by (4.4), we can get that boundary value problem (1.1) has at least one positive solution in $P \cap \Omega_{1}$. Then boundary value problem (1.1) with $\lambda \geq \lambda^{*}$ and $0 \leq a \leq a_{0}$ has at least three positive solutions in $P \cap \Omega_{3}$.

Similar to the proof of Theorem 4.3, we can prove the following theorem.

Theorem 4.4 Suppose that $\lambda>0$, $\liminf _{u \rightarrow+\infty} \inf _{t \in\left[\frac{1}{4}, \frac{3}{4}\right]} f\left(t, t^{\beta-2} u\right)=+\infty, f^{0}=0$, and $f^{\infty}=0$ hold. Then there exists a constant $a_{0}>0$ such that boundary value problem (1.1) has at least two positive solutions with $0 \leq a \leq a_{0}$.

Theorem 4.5 If $f_{\infty}>0$, then there exist constants $\lambda^{*}>0$ and $a_{0}>0$ such that boundary value problem with $\lambda \geq \lambda^{*}$ and $a \geq a_{0}$ (1.1) has no positive solution.

Proof Since $f_{\infty}>0$, there exist constants $\eta>0$ and $r_{1}>0$ such that

$$
f\left(t, t^{\beta-2} u\right)>\eta u, \quad t \in\left[\frac{1}{4}, \frac{3}{4}\right] \text {, and } u \in\left[\frac{1}{4} \gamma_{0} r_{1},+\infty\right) .
$$

Let

$$
\begin{aligned}
& \lambda^{*}=8\left(\left(m_{1}-m_{0}\right)(\beta-1) \eta \gamma_{0} \int_{\frac{1}{4}}^{\frac{3}{4}} g_{1}(s) \mathrm{d} s\right)^{-1}, \\
& a_{0}=2\left(1-m_{1}\right) r_{1}\left(m_{2}+1-2 m_{1}\right)^{-1} .
\end{aligned}
$$

If $u$ is a positive solution of boundary value problem (1.1) with $\lambda \geq \lambda^{*}$ and $a \geq a_{0}$, we will show that this leads to a contradiction. 
In fact, since $T u=u$, we have

$$
\begin{aligned}
\left(\frac{1}{2}\right)^{2-\beta} u\left(\frac{1}{2}\right) & =\lambda\left(\frac{1}{2}\right)^{2-\beta} \int_{0}^{1} G\left(\frac{1}{2}, s\right) f(s, u(s)) \mathrm{d} s+\frac{a\left(\frac{1}{2} m_{2}+\frac{1}{2}-m_{1}\right)}{1-m_{1}} \\
& >\frac{a\left(\frac{1}{2} m_{2}+\frac{1}{2}-m_{1}\right)}{1-m_{1}} \\
& \geq \frac{a_{0}\left(\frac{1}{2} m_{2}+\frac{1}{2}-m_{1}\right)}{1-m_{1}} \\
& =r_{1} .
\end{aligned}
$$

Hence, $\|u\|>r_{1}$.

Because $u \in P$, by (4.5), we can get that

$$
t^{2-\beta} u(t) \geq \gamma_{0} t\|u\|>\frac{1}{4} \gamma_{0} r_{1}, \quad t \in\left[\frac{1}{4}, \frac{3}{4}\right],
$$

and

$$
\begin{aligned}
\left(\frac{1}{2}\right)^{2-\beta} u\left(\frac{1}{2}\right) & =\lambda\left(\frac{1}{2}\right)^{2-\beta} \int_{0}^{1} G\left(\frac{1}{2}, s\right) f(s, u(s)) \mathrm{d} s+\frac{a\left(\frac{1}{2} m_{2}+\frac{1}{2}-m_{1}\right)}{1-m_{1}} \\
& >\frac{1}{8} \lambda^{*}(\beta-1)\left(m_{1}-m_{0}\right) \gamma_{0} \eta\|u\| \int_{\frac{1}{4}}^{\frac{3}{4}} g_{1}(s) \mathrm{d} s+r_{1} \\
& =\|u\|+r_{1} .
\end{aligned}
$$

That is, $\|u\|>\|u\|+r_{1}$, which is a contradiction. Therefore, boundary value problem (1.1) with $\lambda \geq \lambda^{*}$ and $a \geq a_{0}$ has no positive solution.

\section{Illustration}

To illustrate our main results, we present the following examples.

Example 5.1 Consider the boundary value problem

$$
\left\{\begin{array}{l}
D_{0^{+}}^{\frac{1}{4}}\left(\frac{1}{t+1} D_{0^{+}}^{\frac{7}{4}} u(t)\right)+\lambda\left(t^{2}+1\right)\left(160 t^{\frac{1}{4}} u(t)-\frac{51,199}{320} \sin \left(t^{\frac{1}{4}} u(t)\right)\right)=0, \quad t \in(0,1), \\
\lim _{t \rightarrow 0^{+}} t^{\frac{1}{4}} u(t)=a, \quad u(1)=\frac{1}{2} u\left(\frac{1}{4}\right)+\frac{1}{2} u\left(\frac{3}{4}\right), \\
\lim _{t \rightarrow 0^{+}} \frac{1}{(t+1) t^{\frac{3}{4}}} D_{0^{+}}^{\frac{7}{4}} u(t)=0
\end{array}\right.
$$

where $\alpha=\frac{1}{4}, \beta=\frac{7}{4}, f(t, u)=\left(t^{2}+1\right)\left(160 t^{\frac{1}{4}} u-\frac{51,199}{320} \sin \left(t^{\frac{1}{4}} u\right)\right), p(t)=\frac{1}{t+1}>0$, and

$$
A(t)= \begin{cases}0, & 0 \leq t<\frac{1}{4}, \\ \frac{1}{2}, & \frac{1}{4} \leq t<\frac{3}{4}, \\ 1, & \frac{3}{4} \leq t<1 .\end{cases}
$$

Hence,

$$
0<\int_{0}^{1} s^{\beta-1} \mathrm{~d} A(s) \approx 0.57974<1, \quad \gamma_{0} \approx 0.105123<1,
$$




$$
\begin{aligned}
& m_{0} \approx 0.346417, \quad m_{1} \approx 0.57974, \quad m_{2} \approx 1.24439 \\
& f^{0}=\frac{1}{160}<\xi=\frac{1}{80}, \quad f_{\infty}=170>\eta=160 .
\end{aligned}
$$

We can obtain the following results.

(1) It is easy to check that all the conditions of Theorem 3.1 are satisfied. By Theorem 3.1, for each $\lambda$ with $2.75654 \leq \lambda \leq 62.0449$, let a constant $r_{1}=0.01$, then for each $a$ satisfying $0 \leq a \leq 0.00632303-0.00010191 \lambda$, boundary value problem (5.1) has at least one positive solution.

(2) It is easy to see that all the conditions of Theorem 4.5 are satisfied. By Theorem 4.5, let $r_{1}=381$, for all $\lambda \geq 5.51308$ and $a \geq 295.175$, boundary value problem (5.1) has no positive solution.

Example 5.2 Consider the boundary value problem

$$
\left\{\begin{array}{l}
D_{0^{+}}^{\frac{1}{2}}\left((t+1) D_{0^{+}}^{\frac{3}{2}} u(t)\right)+\lambda\left(\mathrm{e}^{t u^{2}(t)}+t \sin \left(t^{\frac{1}{2}} u(t)\right)\right)=0, \quad t \in(0,1) \\
\lim _{t \rightarrow 0^{+}} t^{\frac{1}{2}} u(t)=a, \quad u(1)=\frac{1}{4} u\left(\frac{1}{4}\right)+\frac{1}{8} u\left(\frac{3}{4}\right), \\
\lim _{t \rightarrow 0^{+}} t^{\frac{1}{2}}(t+1) D_{0^{+}}^{\frac{3}{2}} u(t)=0
\end{array}\right.
$$

where $\alpha=\frac{1}{2}, \beta=\frac{3}{2}, f(t, u)=\left(\mathrm{e}^{t u^{2}}+t \sin \left(t^{\frac{1}{2}} u\right)\right), p(t)=t+1>0$ for any $t \in[0,1]$, and

$$
A(t)= \begin{cases}0, & 0 \leq t<\frac{1}{4} \\ \frac{1}{4}, & \frac{1}{4} \leq t<\frac{3}{4} \\ \frac{1}{4}+\frac{1}{8}, & \frac{3}{4} \leq t<1 .\end{cases}
$$

Then

$$
\begin{aligned}
& \int_{0}^{1} s^{\beta-1} \mathrm{~d} A(s) \approx 0.233253<1, \quad \gamma_{0} \approx 0.0428085<1, \\
& m_{0} \approx 0.11244, \quad m_{1} \approx 0.233253, \quad m_{2} \approx 0.644338 \\
& f_{\infty}=+\infty, \quad f_{0}=+\infty .
\end{aligned}
$$

All the conditions of Theorem 4.2 are satisfied. By Theorem 4.2, for given $r>1,0<\lambda \leq$ 1.1124 and each $a$ satisfying $0 \leq a \leq 1-0.402948 \lambda$, boundary value problem (5.2) has at least two positive solutions $u_{1}, u_{2}$.

Example 5.3 Consider the boundary value problem

$$
\left\{\begin{array}{l}
D_{0^{+}}^{\frac{1}{2}}\left((t+1) D_{0^{+}}^{\frac{3}{2}} u(t)\right)+\lambda f(t, u(t))=0, \quad t \in(0,1) \\
\lim _{t \rightarrow 0^{+}} t^{\frac{1}{2}} u(t)=a, \quad u(1)=\int_{0}^{1} s u(s) \mathrm{d} s, \\
\lim _{t \rightarrow 0^{+}} t^{\frac{1}{2}}(t+1) D_{0^{+}}^{\frac{3}{2}} u(t)=0
\end{array}\right.
$$


where $\alpha=\frac{1}{2}, \beta=\frac{3}{2}, p(t)=t+1, A(t)=\frac{1}{2} t^{2}$, and

$$
f(t, u)= \begin{cases}t^{2} u^{2}, & 0 \leq t \leq 1 \text { and } 0 \leq u<1 \\ t^{2} \sqrt{u}, & 0 \leq t \leq 1 \text { and } u \geq 1\end{cases}
$$

Then $\liminf \inf _{u \rightarrow+\infty} \inf _{t \in\left[\frac{1}{4}, \frac{3}{4}\right]} f\left(t, t^{\beta-2} u\right)=+\infty, f^{0}=0$, and $f^{\infty}=0$,

$$
\begin{aligned}
& 0<\int_{0}^{1} s^{\beta-1} \mathrm{~d} A(s)=\int_{0}^{1} s^{\frac{3}{2}} \mathrm{~d} s=\frac{2}{5}<1, \\
& m_{0}=\frac{2}{7}, \quad m_{1}=\frac{2}{5}, \quad m_{2}=\frac{2}{3}, \quad \gamma_{0}=\frac{6}{133}<1 .
\end{aligned}
$$

All the conditions of Theorem 4.4 are satisfied. By Theorem 4.4, there exists a constant $a_{0}>0$ such that boundary value problem (5.3) with $0 \leq a \leq a_{0}$ has at least two positive solutions for $\lambda>0$.

\section{Acknowledgements}

This work is supported by the National Natural Science Foundation of China (No. 11171220, 11571207).

Funding

This work is supported by the National Natural Science Foundation of China (No. 11171220 and 11571207).

\section{Competing interests}

The authors declare that they have no competing interests.

\section{Authors' contributions}

The authors declare that the work was realized in collaboration with the same responsibility. All authors read and approved the final manuscript.

\section{Author details}

${ }^{1}$ College of Science, University of Shanghai for Science and Technology, Shanghai, China. ${ }^{2}$ College of Mathematics and System Science, Shandong University of Science and Technology, Qingdao, P.R. China.

\section{Publisher's Note}

Springer Nature remains neutral with regard to jurisdictional claims in published maps and institutional affiliations.

Received: 25 January 2019 Accepted: 6 June 2019 Published online: 09 July 2019

\section{References}

1. Podlubny, I.: Fractional Differential Equations. Academic Press, New York (1999)

2. Diethelm, K.: The Analysis of Fractional Differential Equations. Lecture Notes in Mathematics, vol. 2004. Springer, Heidelberg (2010)

3. Samko, S.G., Kilbas, A.A., Marichev, O.I.: Fractional Integrals and Derivatives. Gordon \& Breach, New York (1993)

4. Kilbas, A.A., Srivastava, H.M., Trujillo, J.J.: Theory and Applications of Fractional Differential Equations. Elsevier, Amsterdam (2006)

5. Song, Q., Bai, Z.: Positive solutions of fractional differential equations involving the Riemann-Stieltjes integral boundary condition. Adv. Differ. Equ. 2018, 183 (2018)

6. Liu, X., Jia, M., Ge, W.: The method of lower and upper solutions for mixed fractional four-point boundary value problem with p-Laplacian operator. Appl. Math. Lett. 65, 56-62 (2017)

7. Liu, X., Jia, M., Ge, W.: Multiple solutions of a $p$-Laplacian model involving a fractional derivative. Adv. Differ. Equ. 2013, $126(2013)$

8. Tian, Y., Sun, S., Bai, Z.: Positive solutions of fractional differential equations with p-Laplacian. J. Funct. Spaces 2017, Article ID 3187492 (2017)

9. Jleli, M., Nieto, J.J., Samet, B.: Lyapunov-type inequalities for a higher order fractional differential equation with fractional integral boundary conditions. Electron. J. Qual. Theory Differ. Equ. 2017, 16 (2017)

10. Dhar, S., Kong, Q., McCabe, M.: Fractional boundary value problems and Lyapunov-type inequalities with fractional integral boundary conditions. Electron. J. Qual. Theory Differ. Equ. 2016, 43 (2016)

11. Jiang, D., Yuan, C.: The positive properties of the Green function for Dirichlet-type boundary value problems of nonlinear fractional differential equations and its application. Nonlinear Anal. 72, 710-719 (2010)

12. Lan, K., Lin, W.: Positive solutions of systems of Caputo fractional differential equations. Commun. Appl. Anal. 17(1), 61-86 (2013) 
13. He, J., Jia, M., Liu, X., et al.: Existence of positive solutions for a high order fractional differential equation integral boundary value problem with changing sign nonlinearity. Adv. Differ. Equ. 2018, 49 (2018)

14. Liu, X., Jia, M.: Solvability and numerical simulations for BVPs of fractional coupled systems involving left and right fractional derivatives. Appl. Math. Comput. 353, 230-242 (2019)

15. Jia, M., Liu, X.: The existence of positive solutions for fractional differential equations with integral and disturbance parameter in boundary conditions. Abstr. Appl. Anal. 2014, 36 (2014)

16. Feng, M., Zhang, X., Ge, W.: New existence results for higher-order nonlinear fractional differential equation with integral boundary conditions. Bound. Value Probl. 2011, Article ID 720702 (2011)

17. Liu, X., Jia, M.: The method of lower and upper solutions for the general boundary value problems of fractional differential equations with $p$-Laplacian. Adv. Differ. Equ. 2018, 28 (2018)

18. Jia, M., Liu, X.: Multiplicity of solutions for integral boundary value problems of fractional differential equations with upper and lower solutions. Appl. Math. Comput. 232(232), 313-323 (2014)

19. Henderson, J., Luca, R.: Positive solutions for a system of semipositone coupled fractional boundary value problems. Bound. Value Probl. 2016(1), 1 (2016)

20. Webb, J.R.L.: Existence of positive solutions for a thermostat model. Nonlinear Anal., Real World Appl. 13(2), 923-938 (2012)

21. Hao, Z., Debnath, L.: On eigenvalue intervals and eigenfunctions of fourth-order singular boundary value problems. Appl. Math. Lett. 18(5), 543-553 (2005)

22. Graef, J.R., Yang, B.: Positive solutions to a multi-point higher order boundary value problem. J. Math. Anal. Appl. 316(1-2), 247-257 (2009)

23. Webb, J.R.L., Infante, G.: Semi-positone nonlocal boundary value problems of arbitrary order. Commun. Pure Appl. Anal. 9(2), 563-581 (2010)

24. Han, Z., Lu, H., Zhang, C.: Positive solutions for eigenvalue problems of fractional differential equation with generalized p-Laplacian. Appl. Math. Comput. 257, 526-536 (2015)

25. Li, L., Li, H., Dai, Q.: Multiple solutions for the eigenvalue problem of nonlinear fractional differential equations. Commun. Math. Res. 32(2), 173-184 (2016)

26. Bai, Z:: Eigenvalue intervals for a class of fractional boundary value problem. Comput. Math. Appl. 64(10), 3253-3257 (2012)

27. Zhang, Y., Gu, Y.: Eigenvalue intervals for nonlocal fractional order differential equations involving derivatives. J. Appl. Math. Comput. 55(1-2), 1-16 (2016)

28. Zhang, X., Liu, L., Wiwatanapataphee, B., et al.: The eigenvalue for a class of singular $p$-Laplacian fractional differential equations involving the Riemann-Stieltjes integral boundary condition. Appl. Math. Comput. 235(4), 412-422 (2014)

29. Zhao, K.: Impulsive integral boundary value problems of the higher-order fractional differential equation with eigenvalue arguments. Adv. Differ. Equ. 2015(1), 1 (2015)

30. Su, X., Jia, M., Fu, X.: On positive solutions of eigenvalue problems for a class of $p$-Laplacian fractional differential equations. J. Appl. Anal. Comput. 8(1), 152-171 (2018)

31. Kong, L., Kong, Q.: Second-order boundary value problems with nonhomogeneous boundary conditions (I). Math. Nachr. 278(1), 173-193 (2005)

32. Kong, L., Kong, Q.: Second-order boundary value problems with nonhomogeneous boundary conditions (II). J. Math. Anal. Appl. 330(2), 1393-1411 (2007)

33. Kong, L., Kong, Q.: Higher order boundary value problems with nonhomogeneous boundary conditions. Nonlinear Anal. 72(1), 240-261 (2010)

34. Su, X., Jia, M., Li, M.: The existence and nonexistence of positive solutions for fractional differential equations with nonhomogeneous boundary conditions. Adv. Differ. Equ. 2016(1), 1 (2016)

35. Wang, W., Guo, X.: Eigenvalue problem for fractional differential equations with nonlinear integral and disturbance parameter in boundary conditions. Bound. Value Probl. 2016(1), 42 (2016)

36. Guo, D., Lakshmikantham, V.: Nonlinear Problems in Abstract Cones. Academic Press, New York (1988)

\section{Submit your manuscript to a SpringerOpen ${ }^{\circ}$ journal and benefit from:}

- Convenient online submission

- Rigorous peer review

- Open access: articles freely available online

- High visibility within the field

Retaining the copyright to your article

Submit your next manuscript at $>$ springeropen.com 